IRA-International Journal of Applied Sciences ISSN 2455-4499; Vol.03, Issue 03 (2016)

\title{
Chemical Reaction Effects on MHD Free Convective Flow through Porous Medium with Constant Suction and Heat Flux
}

\author{
${ }^{1}$ B. Seshaiah, ${ }^{2}$ S.V.K. Varma \\ ${ }^{1}$ Associate Professor, Department of Mathematics, \\ Santhiram Engineering College, Nandyal, India. \\ ${ }^{2}$ Professor, Department of Mathematics, \\ Sri Venkateswara University, Tirupati, India.
}

DOI: http://dx.doi.org/10.21013/jas.v3.n3.p4

\section{How to cite this paper:}

Seshaiah, B., \& Varma, S. (2016). Chemical Reaction Effects on MHD Free Convective Flow through Porous Medium with Constant Suction and Heat Flux. IRA-International Journal of Applied Sciences (ISSN 2455-4499), 3(3). doi:http://dx.doi.org/10.21013/jas.v3.n3.p4

(C) Institute of Research Advances

\section{(cc) EY-NC}

This works is licensed under a Creative Commons Attribution-Non Commercial 4.0 International License subject to proper citation to the publication source of the work.

Disclaimer: The scholarly papers as reviewed and published by the Institute of Research Advances (IRA) are the views and opinions of their respective authors and are not the views or opinions of the IRA. The IRA disclaims of any harm or loss caused due to the published content to any party. 


\begin{abstract}
The Objective of the present study is to investigate to free convection and mass transfer flow of a viscous incompressible and electrically conducting fluid through a porous medium bounded by vertical infinite surface with constant suction velocity and constant heat flux under the action of uniform magnetic field applied normal to the direction of flow.
\end{abstract}

Keywords: Suction, Heat flux, chemical reaction, Conductivity, porous medium, Viscosity.

\title{
I. INTRODUCTION
}

Flows of fluids through porous medium are of principal interest because these are quite prevalent in nature. Such flows have attracted the attention of a number of scholars due to their applications in many branches of Science and technology, viz. in the fields of Agriculture Engineering to study the underground water resources, seepage of water in river beds, in Petroleum technology to study the movement of natural gas, oil and water through the reservoirs, in Chemical Engineering for filtration and purification process.

In the investigation of Singh (2006) has presented a detailed Analysis a Problem on Unsteady free convection and mass transfer flow of an incompressible viscous liquid through a porous medium past an infinite vertical porous plate subject to time dependent suction velocity normal to the plate using perturbation technique. Singh et al (2005) and Singh studied the suction velocity distribution on viscous flow and transfer problems along flat and vertical porous plates.

From the Technical point of view, MHD-free convection flows have also great significance for the application in the fields of Stellar and Planetary Magnetospheres, Aeronautics, Chemical Engineering and Electronics . Cramer and Pai (1975) model studies of the above phenomenon of MHD-free convection flows have been made by many of them such as, Georganlopoulos(1979), Nauousis et al (1980), Nawal Kishore etal (1981), Hady and Hassanien (1986), and Singh and Rana(1992)

The Effect of Chemical reaction depend whether the reaction is homogeneous or heterogeneous, this depends on whether they occur at an interface or a single phase volume reaction. In well-mixed systems the reactions heterogeneous, it takes place at an interface and homogeneous, if it takes place in solution. In most cases of Chemical reactions, the reaction rate depends on the concentration of the species itself.

Chamka (2000) studied the MHD flow past a uniformly stretched vertical permeable surface in the presence of heat generation / absorption and a Chemical reaction, Apelblat(1994) studied analytical solution for Mass transfer with a Chemical reaction order. Das et al (1999) have studied the Effect of homogeneous first order chemical reaction on the flow past an impulsively started 
infinite vertical plate with uniform heat flux and mass transfer. Again Mass transfer effects on moving isothermal vertical plate in the presence of chemical reaction studied by Das et al and Chambre and Young (1990) have analyzed a first order chemical reaction in the neighbourhood of a horizontal plate.

The Effect of variable permeability on combined free and forced convection in porous media was studied by Chandrasekhar and Namboodivi later on mixed convection in porous media adjacent to a vertical uniform heat flux surface was studied by Joshi and Gebhart heat and mass transfer in a porous medium was discussed by Bejan and Khair. The above problem was studied in presence of buoyancy effect by Trevisan and Bejan. Lai and Kulacki studied the effect of variable viscosity on Convective heat flux was investigated by Sharma. The Steady two dimensional flows through a porous medium bounded by a vertical infinite surface with constant suction velocity and constant heat flux in the presence of free convection current was studied by Sharma. Convection in a porous medium with inclined temperature gradient was investigated by Nield. The problem of mixed convection along an isothermal vertical plate in porous medium with injection and suction was studied by Hooper $e t$ al.

The Objective of the present study is to investigate to free convection and mass transfer flow of a viscous incompressible and electrically conducting fluid through a porous medium bounded by vertical infinite surface with constant suction velocity and constant heat flux under the action of uniform magnetic field applied normal to the direction of flow.

\section{NOMENCLATURE}

$C$ : Concentration of Species

$D$ : Chemical molecular diffusivity

$g \quad:$ Acceleration due to gravity

$k$ : Permeability of porous medium

$M \quad$ : Magnetic parameter

$q$ : Constant heat flux

$C_{p}:$ Specific molecular diffusivity

$G_{r}:$ Grashof number for heat transfer

$G_{m}$ : Grashof number for mass transfer

$P_{r} \quad$ : Prandtl number

$S_{c} \quad:$ Schmidt number

$T$ : The fluid temperature

$T_{\infty}$ : The fluid temperature at infinity

$C_{\infty}$ : The species concentration at infinity

$V_{0}:$ Scale of suction velocity

$\lambda$ : Thermal conductivity

$\eta$ : Distance

$B_{0}$ : Magnetic flux density

$K$ : Thermal conductivity 
$N_{u}$ : Nusselt number

$S_{h}:$ Sherewood number

$B^{*}$ : The volumetric coefficient expansion with species concentration

$\beta$ : Coefficient of the volume expansion due to temperature

$\sigma$ : Electrical conductivity

$\rho \quad$ : The density of the fluid

$\mu$ : Fluid dynamic viscosity

$v$ : Fluid Kinematic viscosity

$\theta$ : Dimensionless temperature

$\alpha$ : Permeability parameter

\section{FORMULATION OF THE PROBLEM}

We study two dimensional form of a viscous incompressible electrically conducting fluid through a porous medium occupying semi infinite region of space bounded by a vertical infinite surface, under the action of uniform magnetic field applied normal to the direction of flow. The $\mathrm{x}$-axis is taken normal to it. The fluid properties are assumed to be constant except for the influence of density in the body force term. As the bounding surface is infinite in length, all the variables are functions of $y$ only. hence, by the usual boundary layer approximation the basic equations for steady flow through highly porous medium are:

$\frac{\partial v}{\partial y}=0$

$v \frac{\partial u}{\partial y}=\vartheta \frac{\partial^{2} u}{\partial y^{2}}+g \beta\left(T-T_{\infty}\right)+g \beta^{*}\left(C-C_{\infty}\right)-\left(\frac{\sigma B_{0}^{2}}{\rho}\right) u-\frac{\vartheta}{k} u$

(2)

$v \frac{\partial T}{\partial y}=\left(\frac{\lambda}{\rho C_{p}}\right) \frac{\partial^{2} T}{\partial y^{2}}+\left(\frac{\vartheta}{C_{p}}\right)\left(\frac{\partial u}{\partial y}\right)^{2}$

(3)

$v \frac{\partial C}{\partial y}=D \frac{\partial^{2} C}{\partial y^{2}}=D_{1}\left(C-C_{\infty}\right)$

(4)

The equation of continuity (1) gives

$$
v=\text { constant }=-v_{0}
$$

Where $v_{0}>0$ corresponds to steady suction velocity (normal at the surface).

In view of equation (5), equation (2), (3) and (4) are reduced to

$$
-v_{0} \frac{\partial u}{\partial y}=\vartheta \frac{\partial^{2} u}{\partial y^{2}}+g \beta\left(T-T_{\infty}\right)+g \beta^{*}\left(C-C_{\infty}\right)-\left(\frac{\sigma B_{0}^{2}}{\rho}\right) u-\frac{\vartheta}{k} u
$$

$$
-v_{0} \frac{\partial T}{\partial y}=\left(\frac{\lambda}{\rho C_{p}}\right) \frac{\partial^{2} T}{\partial y^{2}}+\left(\frac{\vartheta}{C_{p}}\right)\left(\frac{\partial u}{\partial y}\right)^{2}
$$


(7)

$-v_{0} \frac{\partial C}{\partial y}=D \frac{\partial^{2} C}{\partial y^{2}}=D_{1}\left(C-C_{\infty}\right)$

(8)

The relevant boundary conditions are

$u=0, \quad T=T_{\infty}, \quad C=C_{\infty}$, for any $y \quad t \leq 0$

$u=0, \quad T_{y}=\frac{-q}{\lambda}, \quad C_{y}=\frac{m}{D}, \quad y=0 \quad t>0$

$u=0, \quad T=T_{\infty}, \quad C=C_{\infty}, \quad y \rightarrow \infty \quad t>0$

(9)

Introducing following non- dimensional parameters into equations (6) (7) \& (8)

$$
\begin{aligned}
& f(\eta)=\frac{u}{v_{0}}, \quad \eta=\frac{v_{0} y}{\vartheta}, \quad P_{r}=\frac{\mu C_{p}}{\lambda}, \quad S_{c}=\frac{\vartheta}{D}, \quad \theta=\frac{\left(T-T_{\infty}\right) \lambda C_{p}}{q \vartheta} \\
& \alpha=\frac{v_{0}^{2} K}{\vartheta^{2}}, C=\frac{\left(C-C_{\infty}\right) v_{0} D}{m \vartheta}, G_{r}=\frac{g \beta q \vartheta^{2}}{v_{0}^{4} \lambda} G_{m}=\frac{g \beta^{*} q \vartheta^{2}}{v_{0}^{4} \lambda}, \\
& M=\frac{\sigma \vartheta B_{0}^{2}}{\rho v_{0}^{2}}, E=\frac{\lambda v_{0}^{3}}{q \vartheta C_{p}}
\end{aligned}
$$

Where $\mathrm{q}$ is the heat flux per unit area and $\mathrm{m}$ is the mass flux per unit area. We get $f^{\prime \prime}+f^{\prime}-f\left(\alpha^{-1}+M\right)=G_{r} \theta-G_{m} C$

$\theta^{\prime \prime}+P_{r} \theta^{\prime}=-P_{r}\left(f^{\prime 2}\right) E$

$C^{\prime \prime}+S_{c} C^{\prime}+S_{c} C=0$

Where the primes denotes differentiation with respect to $\eta$

Corresponding boundary conditions become

$$
\begin{array}{ll}
\eta=0 & f=0, \quad \theta^{\prime}=-1, \quad C^{1}=-1 \\
\eta \rightarrow \infty & f \rightarrow 0, \quad \theta \rightarrow 0, \quad C \rightarrow 0
\end{array}
$$


In order to obtain a solution of above coupled non linear system of equations (11), (12) \& (13) we expand $f, \theta$ and $C$ in powers of Eckert number $\mathrm{E}$ assuming that it is very small, This is justified in low speed incompressible flow. hence we can write

$$
\begin{aligned}
& f(\eta)=f_{0}(\eta)+E f_{1}(\eta)+o\left(E^{2}\right) \\
& \theta(\eta)=\theta_{0}(\eta)+E \theta_{1}(\eta)+o\left(E^{2}\right) \\
& C(\eta)=C_{0}(\eta)+E C_{1}(\eta)+o\left(E^{2}\right)
\end{aligned}
$$

Substituting equation (2.15) into equations (11), (12), (13) equating coefficients of same power of $\mathrm{E}$ and neglecting higher order terms in $\mathrm{E}$, we find

$$
\begin{aligned}
& f_{0}^{\prime \prime}+f_{0}^{\prime}-f_{0}\left(\alpha^{-1}+M\right)=-G_{r} \theta_{0}-G_{m} C_{0} \\
& f_{1}^{\prime \prime}+f_{1}^{\prime}-f_{1}\left(\alpha^{-1}+M\right)=-G_{r} \theta_{1}-G_{m} C_{1} \\
& \theta_{0}^{\prime \prime}+P_{r} \theta_{0}^{\prime}=0 \\
& \theta_{1}^{\prime \prime}+P_{r} \theta_{1}^{\prime}=-P_{r}\left({f_{1}^{\prime}}^{2}\right) \\
& C_{0}^{\prime \prime}+S_{c} C_{0}^{\prime}+K_{r} C_{0}=0 \\
& C_{1}^{\prime \prime}+S_{c} C_{1}^{\prime}+K_{r} C_{1}=0
\end{aligned}
$$

with corresponding boundary conditions

$$
\begin{aligned}
& f_{0}=0, \quad f_{1}=0, \quad \theta_{0}^{\prime}=-1, \quad \theta_{1}^{\prime}=0, \quad C_{0}^{\prime}=-1, \quad C_{1}^{\prime}=0 \text { at } \quad \eta=0 \\
& f_{0} \rightarrow 0, \quad f_{1} \rightarrow 0, \quad \theta_{0} \rightarrow 0, \theta_{1} \rightarrow 0, \quad C_{0} \rightarrow 0, \quad C_{1} \rightarrow 0 \text { at } \quad \eta \rightarrow 0
\end{aligned}
$$

Solving equations (2.16)-(2.21) under boundary conditions (22), we obtain

$$
\begin{aligned}
& f_{0}=K_{4} e^{-m_{8} \eta}-\frac{K_{2}}{P_{r}} e^{-P_{r} \eta}-\frac{K_{3}}{m_{2}} e^{-m_{2} \eta} \\
& f_{1}= \\
& N_{8} e^{-m_{12} \eta}-N_{1} e^{-P_{r} \eta}+N_{2} e^{-2 m_{8} \eta}+N_{3} e^{-2 P_{r} \eta}+N_{4} e^{-2 m_{2} \eta}+N_{5} e^{-\left(m_{8}+P_{r}\right) \eta}+ \\
& N_{6} e^{-\left(m_{2}+P_{r}\right) \eta}+N_{7} e^{-\left(m_{2}+m_{8}\right) \eta}
\end{aligned}
$$


(24)

$\theta_{0}=\frac{1}{P_{r}} e^{-P_{r} \eta}$

$\theta_{1}=a_{14} e^{-P_{r} \eta}-a_{1} e^{-2 m_{8} \eta}+a_{2} e^{-2 P_{r} \eta}-a_{3} e^{-2 m_{2} \eta}-a_{4} e^{-\left(m_{8}+P_{r}\right) \eta}-$

$a_{5} e^{-\left(m_{2}+P_{r}\right) \eta}+a_{6} e^{-\left(m_{2}+m_{8}\right) \eta}$

$C_{0}=\frac{1}{m_{2}} e^{-m_{2} \eta}$

$C_{1}=0$

Substituting the solutions of equations (23) - (28) in (11), we obtain

$$
\begin{aligned}
& f(\eta)=K_{4} e^{-m_{8} \eta}-\frac{K_{2}}{P_{r}} e^{-P_{r} \eta}-\frac{K_{3}}{m_{2}} e^{-m_{2} \eta}+E\left(N_{8} e^{-m_{12} \eta}-N_{1} e^{-P_{r} \eta}+\right. \\
& N_{2} e^{-2 m_{8} \eta}+N_{3} e^{-2 P_{r} \eta}+N_{4} e^{-2 m_{2} \eta}+N_{5} e^{-\left(m_{8}+P_{r}\right) \eta}+N_{6} e^{-\left(m_{2}+P_{r}\right) \eta}+ \\
& \left.N_{7} e^{-\left(m_{2}+m_{8}\right) \eta}\right) \\
& \theta(\eta)=\frac{1}{P_{r}} e^{-P_{r} \eta}+E\left(a_{14} e^{-P_{r} \eta}-a_{1} e^{-2 m_{8} \eta}+a_{2} e^{-2 P_{r} \eta}-a_{3} e^{-2 m_{2} \eta}-\right. \\
& \left.a_{4} e^{-\left(m_{8}+P_{r}\right) \eta}-a_{5} e^{-\left(m_{2}+P_{r}\right) \eta}+a_{6} e^{-\left(m_{2}+m_{8}\right) \eta}\right)
\end{aligned}
$$

$C(\eta)=\frac{1}{m_{2}} e^{-m_{2} \eta}$ 


\section{IV . SKIN-FRICTIONS, NUSSELT NUMBER, SHARE-WOOD NUMBER}

$\underline{\text { Skin-friction coefficient }(\tau)}$ :

$\tau=\left(\frac{\partial f(\eta)}{\partial \eta}\right)_{\eta=0}=\tau_{0}+E\left(\tau_{1}\right)$

Where $\tau_{0}=-K_{4} m_{8}+K_{2}+K_{3}$

$\tau_{1}=m_{12} N_{8}-N_{1} P_{r}-2 N_{2} m_{8}-2 N_{3} P_{r}-2 N_{4} m_{2}-N_{5}\left(P_{r}+m_{8}\right)-$

$N_{6}\left(P_{r}+m_{2}\right)-N_{7}\left(m_{2}+m_{8}\right)$

$\underline{\text { Rate of heat transfer in terms of Nusselt number }\left(N_{u}\right)}$

$$
\begin{aligned}
& N_{u}=\left(\frac{\partial \theta(\eta)}{\partial \eta}\right)_{\eta=0} \\
& -1+E\left(-a_{13} P_{r}+2 m_{8} a_{1}+2 P_{r} a_{2}+2 m_{2} a_{3}+a_{4}\left(P_{r}+m_{8}\right)+a_{5}\left(P_{r}+m_{2}\right)+\right. \\
& \left.a_{6}\left(m_{2}+m_{8}\right)\right)
\end{aligned}
$$

The rate of mass transfer in terms of Share-wood number $\left(S_{h}\right)$

$$
S_{h}=\left(\frac{\partial C(\eta)}{\partial \eta}\right)_{\eta=0}=-1
$$

\section{RESULTS AND DISCUSSION:}

Numerical calculations have been carried out for different values of Prandtl number $\left(P_{r}\right)$, Magnetic parameter $(M)$, Grashoff number for heat transfer $\left(G_{r}\right)$, Grashof number for mass transfer $\left(G_{m}\right)$, Permeability parameter $(\alpha)$ Chemical reaction parameter $\left(K_{r}\right)$, Schmidt number $\left(S_{c}\right)$. Numerical results for the velocity, temperature and concentration fields are displayed through the figs (1) - (10) and tables.

Fig (1) \& (2) displays the effects of Chemical reaction parameter $\left(K_{r}\right)$ and Eckert number $\left(E_{c}\right)$ on the velocity field for the cases cooling and heating of the plates respectively. In the case of cooling of the plate the velocity increases near the plate attains the maximum velocity at $\eta=0.05$ and then decreases rapidly for 
$\eta>0.1$. Also noticed that the velocity increases with decreases Chemical reaction parameter $\left(K_{r}\right)$ values and the velocity increases with an increase in Eckert number $\left(E_{c}\right)$. In fig. (2) the opposite phenomenon observed.

Fig (3) \& (4) displays the effects of modified Grashoff number $\left(G_{r}\right)$ and magnetic parameter $(M)$ on the velocity field for the cases of externally cooling and heating of plates. It is observed that from fig (3) the velocity increases with an increase in the values of $\left(G_{m}\right)$. Also it is noticed that the velocity increases near the plate attains the maximum velocity at $\eta=0.1$ and then decreases tends to zero as $\eta \rightarrow \infty$.for increasing values of magnetic parameter $M$ the velocity decreases. The opposite phenomenon is observed from fig (4).

Fig (5) displays the effect of permeability parameter $(\alpha)$ on the temperature field. It is noticed that the temperature decreases with an increase in $\alpha$ values.

The effect of Schmidt number $S_{c}$ on the temperature profiles shows in fig (6). It is noticed that the temperature field decreases with an increase in Schmidt number values

Temperature profiles for various values of Grashoff number for mass $\operatorname{transfer}\left(G_{r}\right)$ are shown in fig (7).It is observed that the Temperature increases with an increase in $\left(G_{r}\right)$.

The effect of Grashoff number $G_{m}$ on the temperature profiles shows in fig (8). It is noticed that the temperature an increases with an increase in $G_{m}$ values

Fig (9) shows the effect of Chemical reaction parameter $K_{r}$ for various values on concentration profiles .It shows the decreases the concentration with an increase in $K_{r}$ values.

Concentration profiles for various values of Schmidt number $S_{c}$ are shown in fig (10). It is shows that the concentration increases with an increase in Schmidt number $S_{c}$.

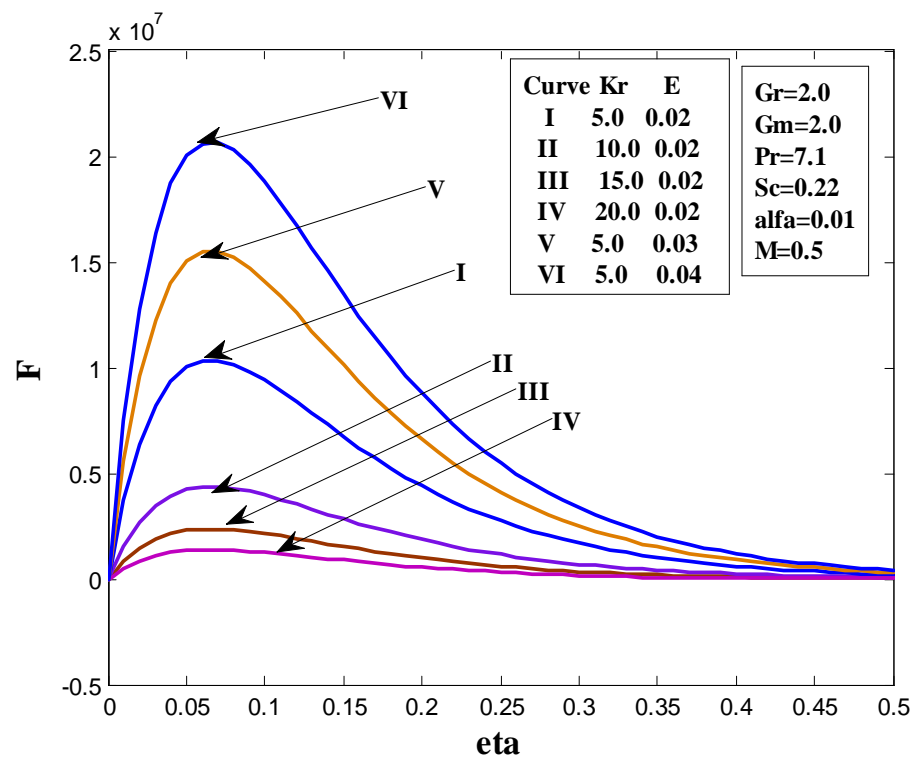

Fig 1: The Effect of $K_{r}$ and $E_{c}$ parameters on Velocity for externally cooled plate 


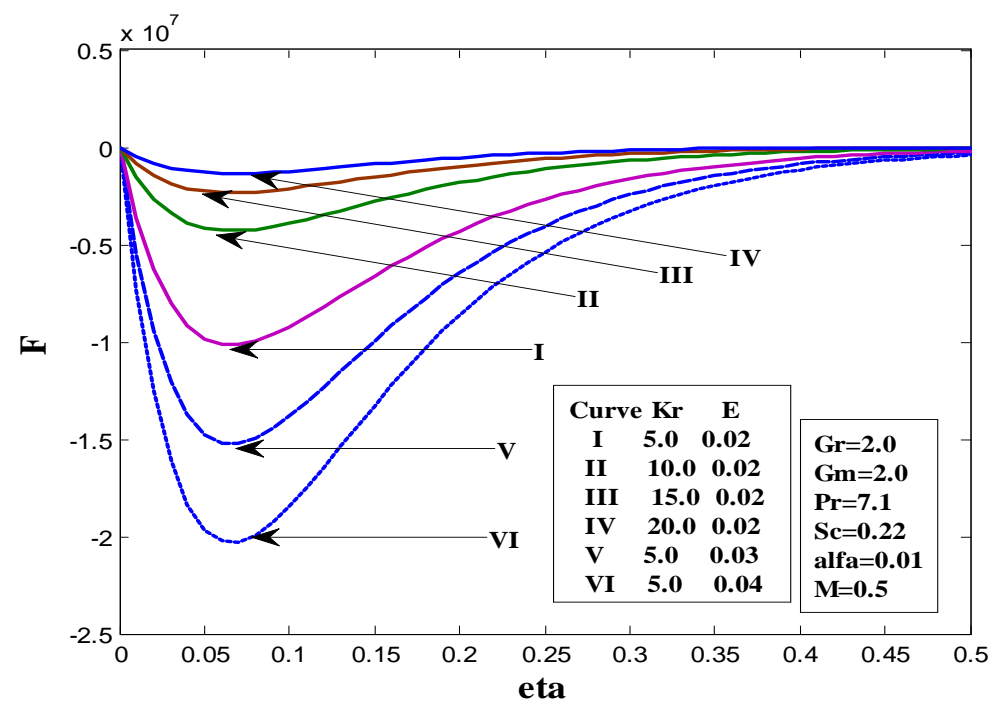

Fig 2: The Effect of $\mathrm{K}_{\mathrm{r}}$ and $\mathrm{E}_{\mathrm{c}}$ parameters on Velocity for externally heated plate

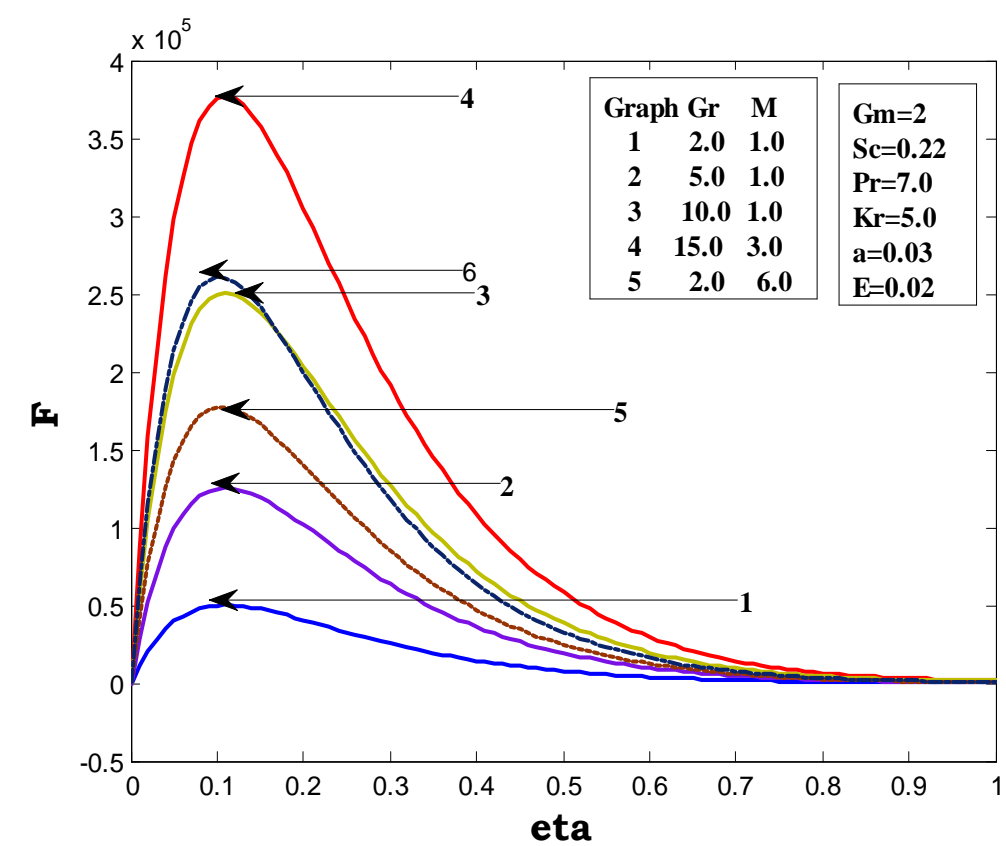

Fig 3: The Effect of $G_{r}$ and $M$ parameters on Velocity for externally cooled plate 


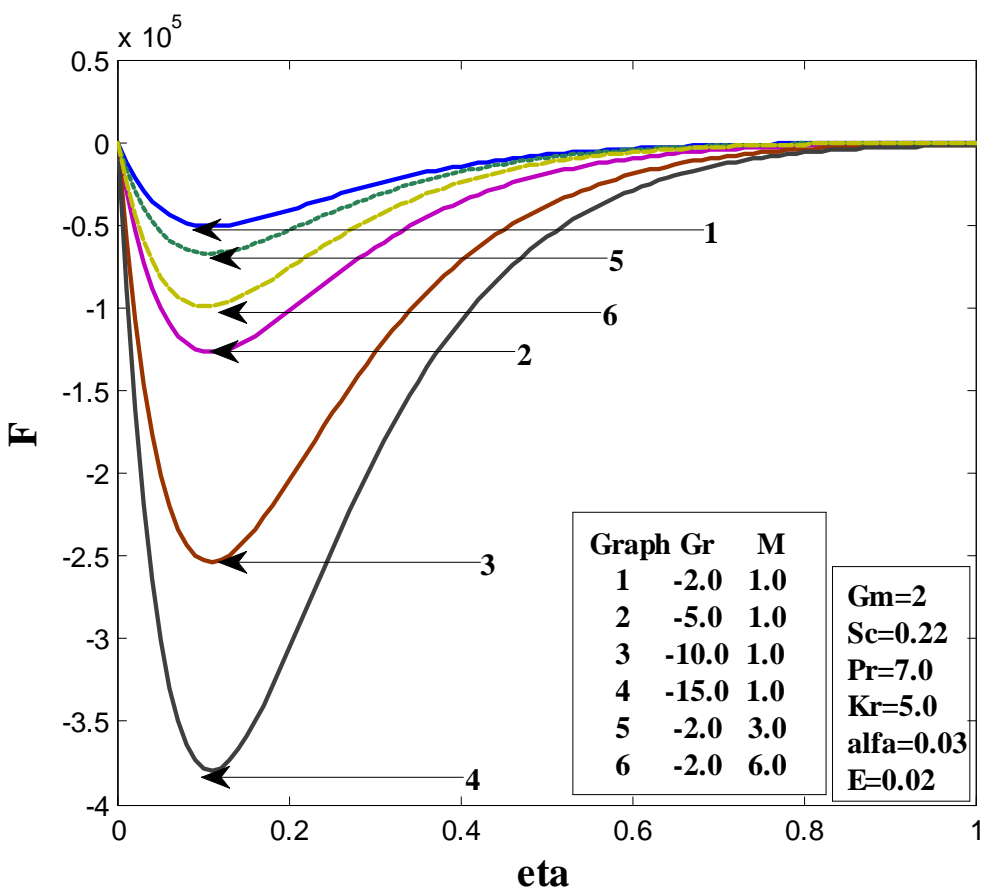

Fig 4: The Effect of $G_{r}$ and $M$ parameters on Velocity for externally heated plate

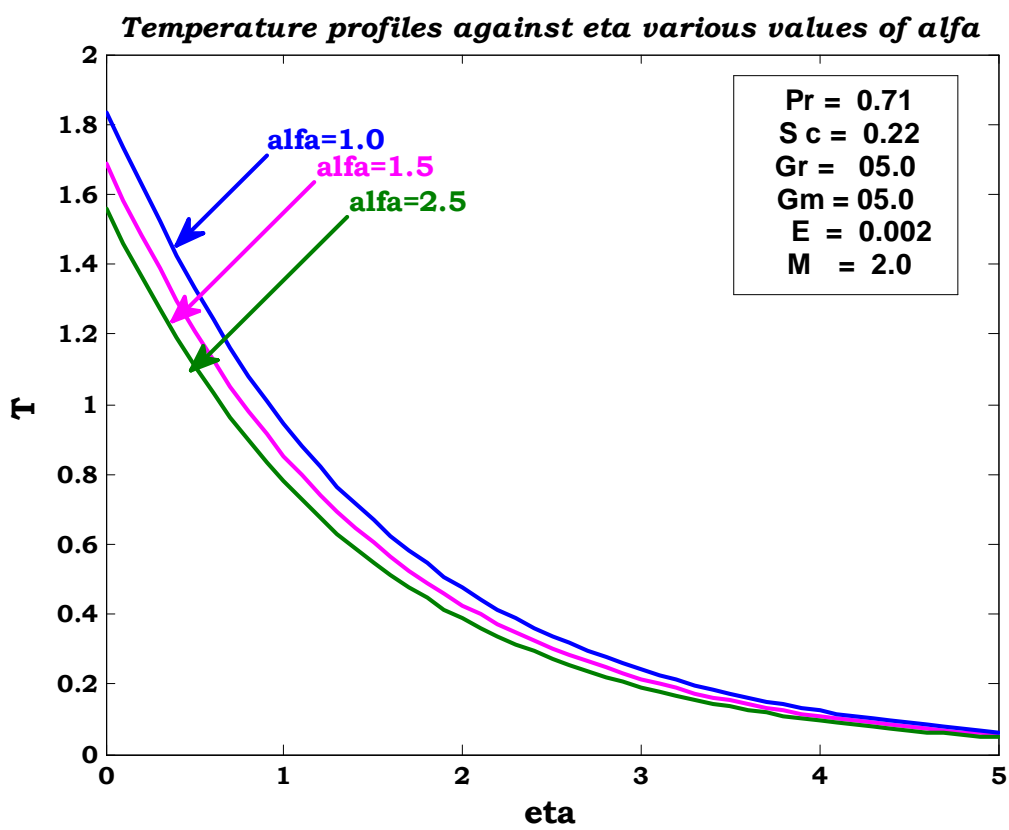

Fig 5: The Effect of permeability parameter $\alpha$ on Temperature 


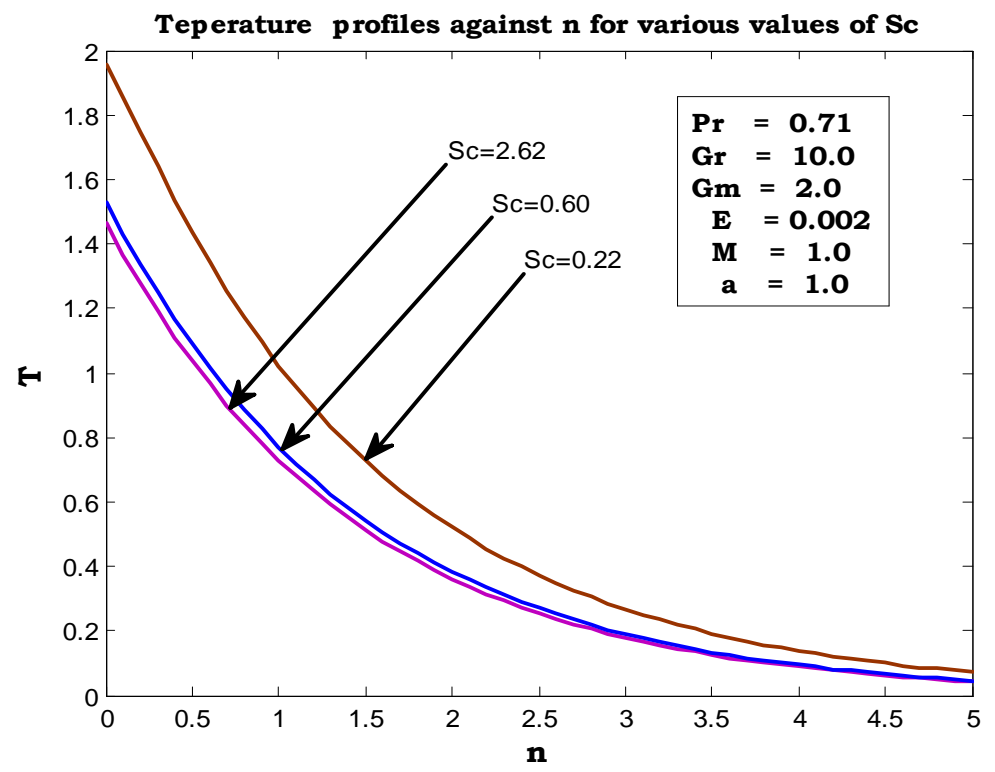

Fig 6: The Effect of Schmidt numberS ${ }_{c}$ on Temperature

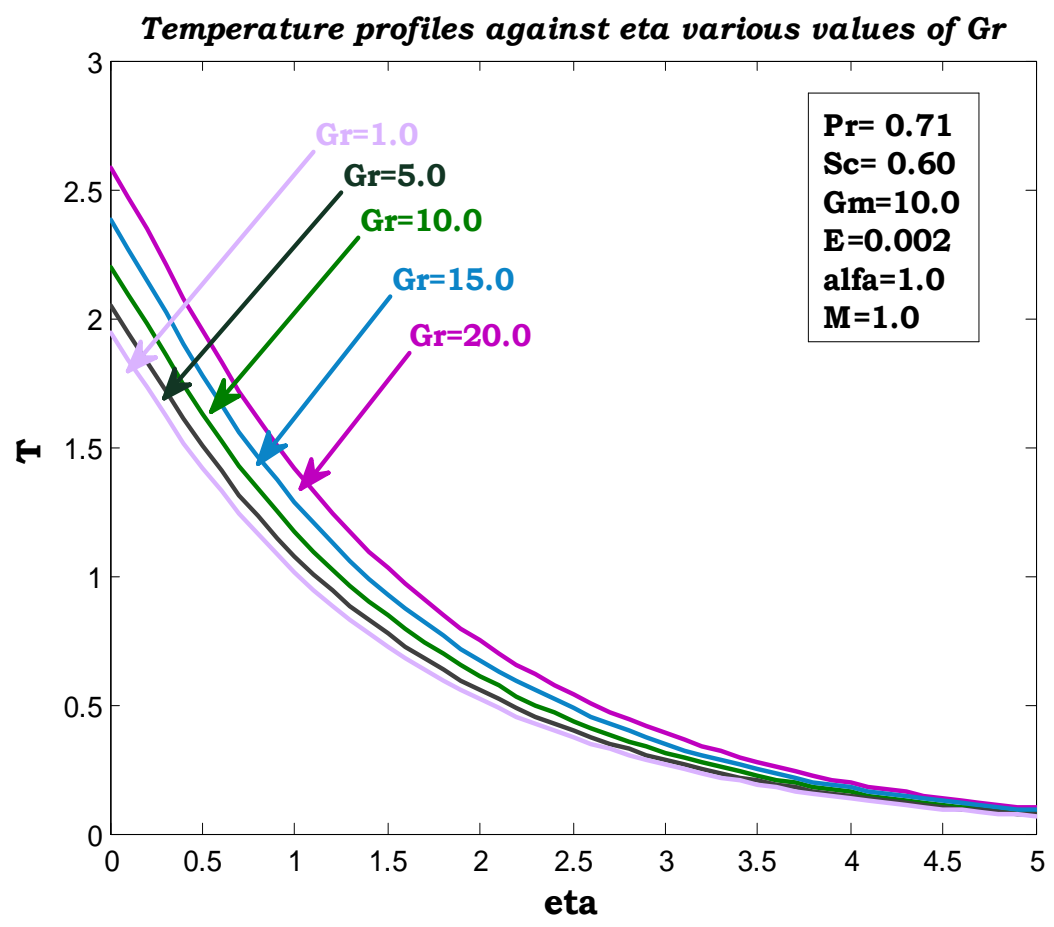

Fig 7: The Effect of Grashoff number for mass transfer $G_{m}$ on Temperature profile 


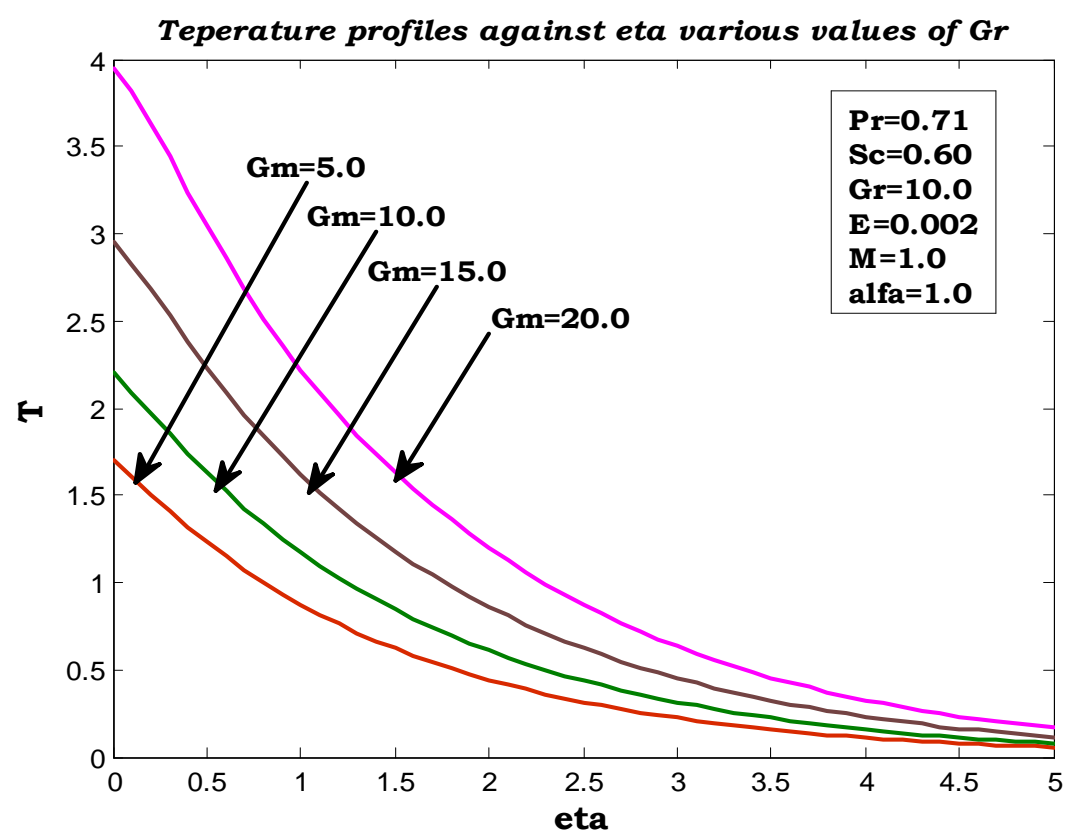

Fig 8: The Effect of Grashoff number for heat transfer $G_{r}$ on Temperature profile

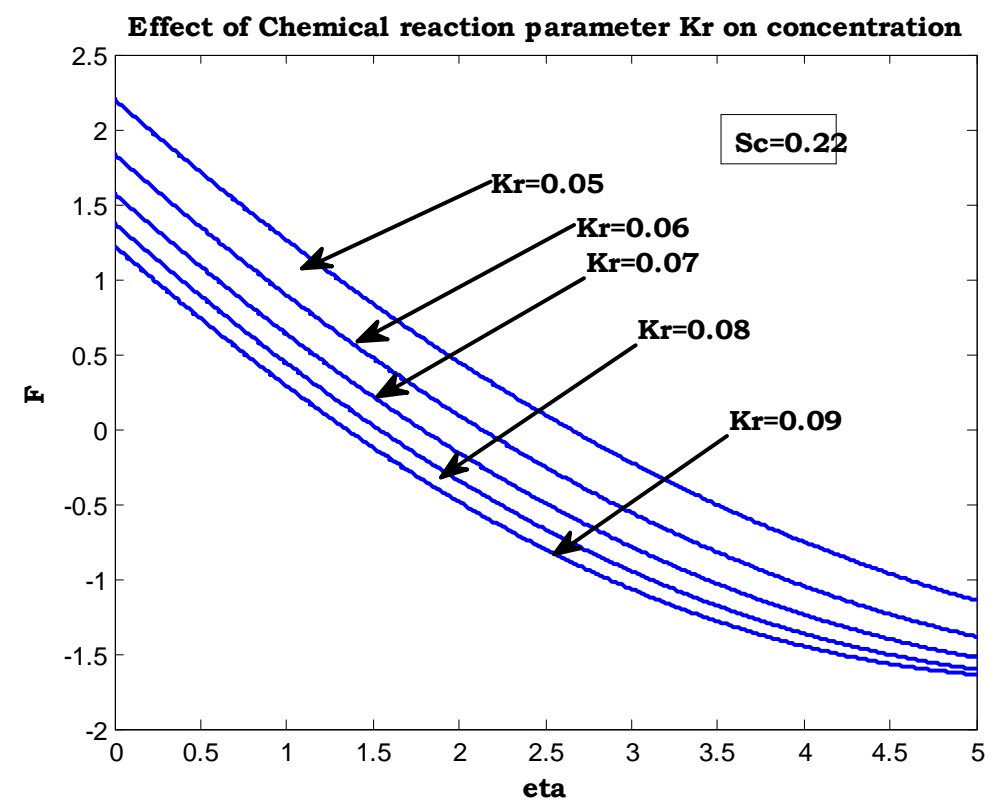

Fig 9: The Effect of Chemical reaction parameter $\mathrm{K}_{\mathrm{r}}$ on Concentration 


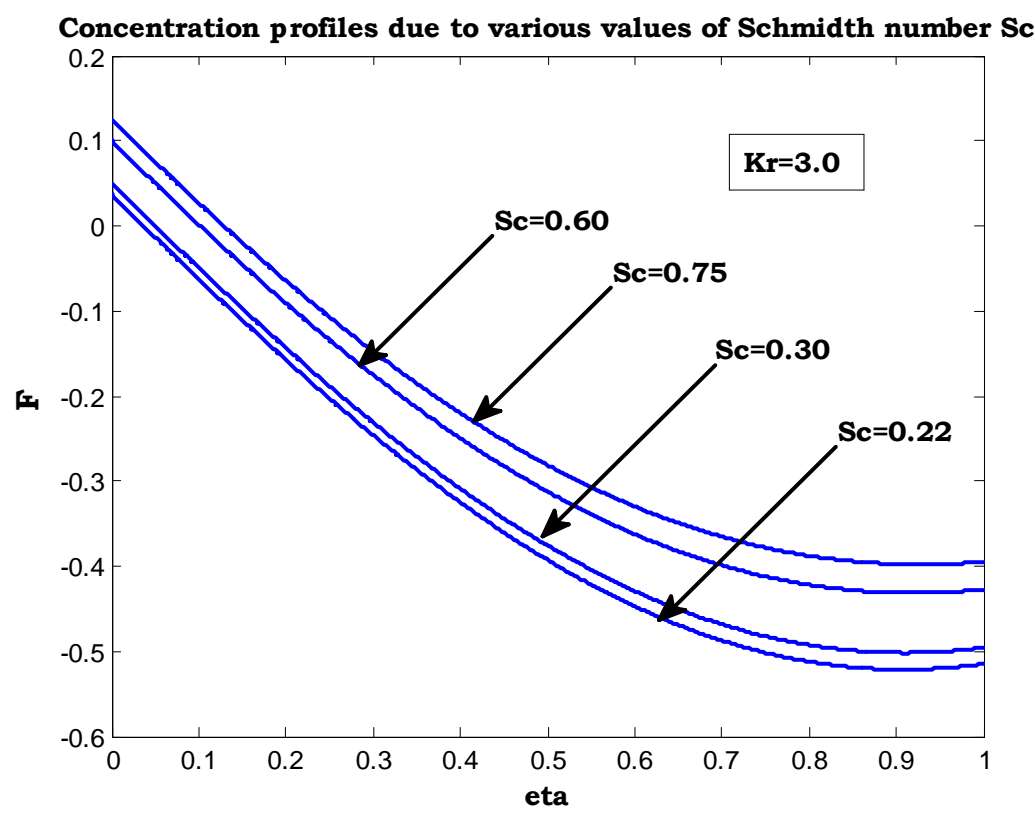

Fig 10: The Effect of Schmidt number $S_{c}$ on Concentration

\section{TABLE-I}

The Skin friction coefficient $(\tau)$ due to cooling of the plate at $\varepsilon=0.005$

\begin{tabular}{|c|c|c|c|c|c|c|c|}
\hline$G_{r}$ & $G_{m}$ & $M$ & $K_{r}$ & $P_{r}$ & $S_{c}$ & $\alpha$ & $\tau$ \\
\hline 2.0 & 2.0 & 0.5 & 0.08 & 0.71 & 0.60 & 3.0 & 7.2136 \\
\hline 5.0 & 2.0 & 0.5 & 0.08 & 0.71 & 0.60 & 3.0 & 19.1928 \\
\hline 2.0 & 5.0 & 0.5 & 0.08 & 0.71 & 0.60 & 3.0 & 14.5124 \\
\hline 2.0 & 2.0 & 1.0 & 0.08 & 0.71 & 0.60 & 3.0 & 5.4070 \\
\hline 2.0 & 2.0 & 0.5 & 0.09 & 0.71 & 0.60 & 3.0 & 7.3787 \\
\hline 2.0 & 2.0 & 0.5 & 0.08 & 7.0 & 0.60 & 3.0 & 4.0398 \\
\hline 2.0 & 2.0 & 0.5 & 0.08 & 0.71 & 0.78 & 3.0 & 10.5404 \\
\hline 2.0 & 2.0 & 0.5 & 0.08 & 0.71 & 0.60 & 4.0 & 7.7482 \\
\hline
\end{tabular}




\section{TABLE-II}

The Skin friction coefficient $(\tau)$ due to heating of the plate at $\varepsilon=0.005$

\begin{tabular}{|c|c|c|c|c|c|c|c|}
\hline$G_{r}$ & $G_{m}$ & $M$ & $K_{r}$ & $P_{r}$ & $S_{c}$ & $\alpha$ & $\tau$ \\
\hline-2.0 & -2.0 & 0.5 & 0.08 & 0.71 & 0.60 & 3.0 & -7.7787 \\
\hline-5.0 & -2.0 & 0.5 & 0.08 & 0.71 & 0.60 & 3.0 & -20.1760 \\
\hline-2.0 & -5.0 & 0.5 & 0.08 & 0.71 & 0.60 & 3.0 & -16.8522 \\
\hline-2.0 & -2.0 & 1.0 & 0.08 & 0.71 & 0.60 & 3.0 & -5.8209 \\
\hline-2.0 & -2.0 & 0.5 & 0.09 & 0.71 & 0.60 & 3.0 & -7.9284 \\
\hline-2.0 & -2.0 & 0.5 & 0.08 & 7.0 & 0.60 & 3.0 & -3.3178 \\
\hline-2.0 & -2.0 & 0.5 & 0.08 & 0.71 & 0.78 & 3.0 & -11.9789 \\
\hline-2.0 & -2.0 & 0.5 & 0.08 & 0.71 & 0.60 & 4.0 & -8.3558 \\
\hline
\end{tabular}

\section{REFERENCES}

[1] N.P. Singh, Ajay Kumar, Arvind Kumar and Atul Kumar Singh(2006) , Heat and mass transfer flow of a viscous fluid past an infinite porous plate with time dependent suction and heat flux, Ultra science Vol.18(3), pp.561-568.

[2] Singh and Ajay Kumar. Int.Comm. Heat Mass Transfer, (2005) 32, pp.1420-1429.

[3] Cramer, K.R. and Pai, S.L(1975) Magnetofluid-dynamics for Engineers and Applied Physicist, Mc Graw-Hill, New York.

[4] Georganlopoulos, G. A(1979).. Astophys. Space Sci.

[5] Nauousis, N.D, Georganlopoulos G.A. and Papaionnon, A.I(1980). Astrophys. Space Science

[6] Naval Kishore, Subhash Tejpal, and H.R. Katiyar Indian (1981), J. pure Appl. Math., pp:Vol 21(1)1211-1372.

[7] Hady , F.M and Hassanien, I.A(1986),Heat and mass transfer for power-law fluids, Indian j. pure Appl. Math., vol.17(1),pp:108-120.

[8] Singh, K.D. and Rana, S.K. Indian (1992), j. pure Appl. Math., Vol 8(2),pp:237- 905.

[9] Chamkha, A. J(2000), Thermal radiation and buoyancy effects on hydro magnetic flow over an accelerating permeable surface with heat source or sink, Int. J. Heat mass transfer, vol.38,pp:1699-1712.

[10] Apelblat(1994), Effects of mass transfer on flow past an impulsively started infinite vertical plate with constant heat flux and chemical reaction, for schung in ingenieurwsen, vol. 60,pp.284-287.

[11] Das, U. N., Dekha, R.K. and Soundalgekar, V.M(1999). Effects of mass transfer on flow past an impulsively started infinite vertical plate with constant heat flux and chemical reaction, The Bulletin, GUMA, vol.5, pp:13-20.

[12] Chambre, P.L. and Young, J. D(1990), On the diffusion of chemical reactive species in a laminar boundary layer flow, The Physics of fluids, Vol.1,pp:48-54. 


\section{APPENDIX}

$$
\begin{aligned}
& k_{1}=\alpha^{-1}+M \quad, a_{7}=\frac{1+k_{5}^{2}}{P_{r}}+\frac{2 k_{7}^{2}}{k_{3}-P_{r}}+\frac{k_{6}^{2}}{2 S_{c}-P_{r}}+\frac{2 k_{7} k_{5}}{k_{3}}+\frac{2 k_{5} k_{6}}{S_{c}} k+\frac{2 k_{7} k_{6}}{k_{3}+S_{c}-P_{r}}, \\
& k_{9}=\frac{1+\sqrt{1+4 k_{1}}}{2} \\
& k_{3}=\frac{1+\sqrt{1+4 k_{1}}}{2}, \quad a_{8}=\frac{G_{r} a_{7}}{\left(P_{r}\right)^{2}-\left(P_{r}\right)-k_{1}}, \quad a_{9}=\frac{G_{r} a_{1}}{\left(2 k_{3}\right)^{2}-\left(2 k_{3}\right)-k_{1}}, \quad a_{91}= \\
& \frac{G_{r} a_{2}}{\left(2 P_{r}\right)^{2}-\left(2 P_{r}\right)-k_{1}} \\
& k_{4}=\frac{k_{5}}{P_{r}}+\frac{k_{6}}{S_{c}}, \quad a_{10}=\frac{G_{r} a_{3}}{4 S_{c}^{2}-2 S_{c}-k_{1}}, \quad a_{11}=\frac{G_{r} a_{4}}{\left(k_{3}+P_{r}\right)^{2}-\left(k_{3}+P_{r}\right)-k_{1}}, a_{12}= \\
& \frac{G_{r} a_{5}}{\left(S_{c}+P_{r}\right)^{2}-\left(S_{c}+P_{r}\right)-k_{1}} \\
& k_{5}=\frac{G_{r}}{P_{r}^{2}-P_{r}-k_{1}}, \quad a_{13}=\frac{G_{r} a_{6}}{\left(k_{3}+S_{r}\right)^{2}-\left(k_{3}+S_{r}\right)-k_{1}}, a_{14}=a_{9}+a_{91}+a_{10}+a_{11}+ \\
& a_{12}+a_{13}-a_{8} \\
& k_{6}=\frac{G_{m}}{S_{c}^{2}-S_{c}-k_{1}} \\
& k_{7}=-k_{3} k_{4} \\
& a_{1}=\frac{P_{r} k_{7}^{2}}{4 k_{3}^{2}-2 k_{3} P_{r}} \\
& a_{2}=\frac{k_{5}^{2}}{P_{r}} \\
& a_{3}=\frac{P_{r} k_{6}^{2}}{4 S_{c}^{2}-2 S_{c} P_{r}} \\
& a_{5}=\frac{2 P_{r} k_{7} k_{5}}{k_{3}^{2}+k_{3} P_{r}} \\
& a_{6}=\frac{2 P_{r} k_{6} k_{5}}{S_{c}^{2}+S_{c} P_{r}} \\
& \left(k_{3}^{2}+S_{c}^{2}+2 k_{3} S_{c}\right)-P_{r}\left(k_{3}+S_{c}\right) \\
& x^{2}
\end{aligned}
$$

